\title{
SEND Test System Strain
}

National Cancer Institute

\section{Source}

National Cancer Institute. SEND Test System Strain. NCI Thesaurus. Code C96373.

The vendor-supplied species, strain or substrain designation for the test system under study. It may combine the species, background strain, substrain, and associated genetic modifications as supplied by the vendor. 